\title{
Value of Duke Treadmill Score in Setting Priority of Patients for Coronary Angiography
}

Mohsen Ali Salama, Mohamed Saad Elgamaal, Aymen Elsaeed Sadek, Ahmed Yehia Hafez

Department of Cardiology, Faculty of Medicine, Al-Azhar University

Corresponding author: Ahmed Yehia Hafez, Mobile: (+20)01010849482;

Email: yehiaahmedyehia2018@gmail.com

\begin{abstract}
Background: exercise treadmill test remains a good test in identification of coronary artery disease(CAD).

The Duke Treadmill Score (DTS), shown to predict CAD better than ST segment deviation alone.

Aim of the work: To determine the potential of DTS in setting priority of patients for coronary angiography.

Patients and methods: This was a prospective study that was done from July 2017 to October 2018 and included 150 patients referred to Damannhour Teaching Hospital for evaluation of chest pain. Patients underwent: history, clinical examination, ECGs, Echocardiography, Treadmill exercise ECG with calculation of DTS then coronary angiography that was correlated with DTS and other variables. Results: There were 59 patients(39.3\%)with high DTS and 91patients $(60.7 \%)$ with intermediate DTS. For the intermediate Duke's risk group the result of coronary angiography distributed on normal (26.4\%), single vessel disease (33\%), two vessel disease (22\%), and three vessels disease or left main disease (18.7\%). For high Duke's risk group the result of coronary angiography showed mainly three vessels disease or left main disease (54.2\%), two vessels disease (27.1\%), single vessel disease (6.8\%) and normal vessel (11.9\%), The P value was $<0.001$.Adding patient's risk factors to intermediate DTS group were statistically significant in the diagnosis of CAD ,the $\mathrm{P}$ value was $<0.001$. Conclusion: The DTS is a good predictor for CAD. Intermediate risk DTS group exercise test results should be managed individually according to clinical data.
\end{abstract}

Keywords: Coronary artery disease; Exercise ECG Stress testing; Duke Treadmill Score; Coronary angiography.

\section{INTRODUCTION}

Nowadays, coronary artery disease (CAD) is the leading cause of death all over the world. Approximately $50 \%$ of all deaths occur due to CAD. Hence, it is important to diagnose patients with CAD ${ }^{(1-3)}$. Despite the numerous advances in imaging technology within recent years, coronary angiography remains the definitive examination for establishing the presence, site and severity of CAD in living patients ${ }^{(4)}$.

Because of the invasiveness and costs of coronary angiography, which prevent its application on a large scale. Coronary atherosclerosis is commonly assessed indirectly by means of noninvasive less expensive laboratory tests ${ }^{(5)}$.

The exercise treadmill test remains a good test in identification of coronary artery disease ${ }^{(\mathbf{6})}$.

The low sensitivity and specificity of exercise electrocardiogram stress testing for diagnosis of coronary artery disease have stimulated increased use and development of noninvasive stress imaging tests ${ }^{(7)}$.

In 1991, Mark and coauthors ${ }^{(8)}$ described a prognostic exercise treadmill score (Duke's score) that was depend on the duration of exercise, degree of STsegment deviation in $\mathrm{mm}$ (depression or elevation), and the presence and severity of chest pain during exercise. This treadmill score has been shown to stratify prognosis accurately for both inpatient and outpatient coronary artery disease populations ${ }^{(9)}$.

The Duke Treadmill Score (DTS), traditionally a prognostic tool, was recently used as a diagnostic tool and shown to predict coronary artery disease better than the ST segment deviation alone ${ }^{(\mathbf{1 0})}$.

aim of the work: To determine the potential of DTS in setting priority of patients for coronary angiography.

\section{PATIENTS AND METHODS}

This was a prospective observation study that was done from July 2017 to October 2018 and included 150 patients referred to Cardiology Department at Damannhour Teaching Hospital for evaluation of chest pain. The study was approved by the Ethics Board of Al-Azhar University and an informed written consent was taken from each participant in the study.

\section{Exclusion criteria:}

1. Prior cardiac catheterization.

2. Revascularization procedure.

3. Resting ST segment or T wave abnormalities such as left bundle branch block, Wolff-Parkinson White or left ventricular hypertrophy in the baseline 12-lead ECG that might interfere with the interpretation of the exercise ECG stress.

4. Contraindications to exercise ECG testing:

a. Acute myocardial infarction $(<2 \mathrm{~d})$.

b. Unstable angina with recent rest pain.

c. Untreated life-threatening cardiac arrhythmias.

d. Advanced atrioventricular block.

e. Acute myocarditis or pericarditis.

f. Critical aortic stenosis.

g. Severe hypertrophic obstructive cardiomyopathy.

h. Uncontrolled hypertension.

i. Acute systemic illness (pulmonary embolism, aortic dissection).

\section{METHODS}

\section{1- History:}

All the patients were subjected to the following:

With a special emphasis on age, gender and major documented risk factors for coronary artery disease or any cardiac condition, Symptoms, number of episodes 
per week, drug history, family history, smoking and history of chronic disease.

2- Clinical examination:

Including general and local examination including blood pressure, pulse, temperature, respiratory rate and, local examination of the heart and lung and other systems.

3- ECG:

All of the patients in this study will undergo resting ECG.

4- ECHO:

All of the patients in this study will undergo ECHO.

We assessed: Systolic and diastolic function, Wall motion and dimensions and Valvular function.

\section{5- Treadmill exercise ECG:}

Exercise test was done for all patients using a continuous progressive maximum protocol (Bruce protocol ${ }^{(11)}$, in which speed and grade were increased every 3 minutes till the end of protocol. 12-lead ECG was done before exercise, at the end of each stage, and at peak exercise The blood pressure was recorded immediately before exercise at the end of each stage and at peak exercise.

\section{Patients' preparation for exercise ECG Stress test included the following:}

1. The patient should not eat or smoke for 3 hours before testing. The patient should wear comfortable shoes. The patient should avoid Unusual physical exertion for at least $12 \mathrm{~h}$ before testing.

2. Cessation of medications, since some drugs interfere with exercise responses, complicating interpretation of exercise testing (e.g. Beta blockers $48 \mathrm{hrs}$ before exercise, diuretic "2 weeks before exercise", digitalis for 1 to 2 weeks before exercise).

3. A detailed explanation of testing procedure should be given, outlining risk and complications.

4. Adequate skin preparation was essential for highquality recordings.

The end point of the test was determined by the following:

1. Limiting symptoms (chest pain, dyspnea, fatigue).

2. Abnormalities of rhythm:

- Ventricular bigeminy or runs of ventriculartachycardia.

- Persistent supraventricular tachycardia.

3. Abnormalities of blood pressure response.

- Decrease in systolic blood pressure more than 10 $\mathrm{mmHg}$ from baseline in spite of an increase in work load.

- Exercise elevation in diastolic blood pressure more than $20 \mathrm{mmHg}$ above patient's resting diastolic blood pressure or elevation in systolic blood pressure $>260$ $\mathrm{mmHg}$.

4. Signs of peripheral circulatory insufficiency, pallor, cyanosis and cold clammy sweating.

The following data were recorded during exercise:

1. ST segment deviation.

2. Anginal pain during exercise.

3. Duration of exercise.

4. Reason for termination of exercise.

5. Peak heart rate achieved during exercise.
6. Total METS achieved.

Duke Treadmill Score: The duke treadmill score was calculated as follow:

Duke Treadmill Score $=$ Exercise time $-(5 \mathrm{x}$

ST deviation) - (4 $\mathrm{x}$ treadmill angina).

- The time of exercise is measured in minutes.

- ST deviation is the largest net deviation, either depression or elevation in any lead except (a VR) (The ECG criterion for positive test is $1 \mathrm{~mm}$ or more of exercise-induced ST-segment depression or elevation at 0.06 after the $\mathrm{J}$ point, relative to the PR segment).

- Treadmill angina is graded on the following scale:

$0=$ no angina during exercise.

$1=$ non limiting angina during exercise.

$2=$ exercise-limiting angina.

In this study, we used a subgrouping system for the treadmill score (DTS): Moderate risk

(DTS

score between 5 and 11)

High risk (DTS score $\leq-11$ )

6- Coronary angiography:

Coronary angiography was done to all patients within 90 days of exercise treadmill testing, utilizing the retrograde, percutaneous transfemoral technique (Judkin's technique).

Coronary lesions were graded with respect to the degree of luminal narrowing. Calipers were used to grade the degree of obstruction, and changes were recorded according to a percent luminal narrowing.

Significant disease was arbitrarily determined to be $70 \%$ or greater luminal diameter narrowing. For the left main coronary artery (LM) $50 \%$ or greater stenosis was considered significant. . This was based on classification of Miranda et al. ${ }^{(12)}$.

\section{Statistics:}

Data were analyzed via Statistical program for social science (SPSS) version 20.0. Quantitative data were expressed as mean \pm standard deviation (SD) while qualitative data were expressed as and percentage frequency. Independent samples t-test of significance was used when comparing between two means. Chisquare $(\chi 2)$ test of significance was used in order to compare proportions between two qualitative parameters. $\mathrm{P}$ value $<0.05$ was considered to be statistically significant.

\section{RESULTS}

There were 150 patients who had positive exercise ECG stress test included in the study.

The patients were divided into two groups according to duke treadmill score, group I (intermediate risk DTS) and group II (high risk DTS)

\section{Demographic data:}

Regarding age; there was statistically significant difference found between two groups the $\mathrm{p}$ value was 0.046. Regarding the sex; there was statistically significant difference found between two group the $p$ value was 0.002 (Table1). 
Table (1): Comparison between the two studied groups according to demographic

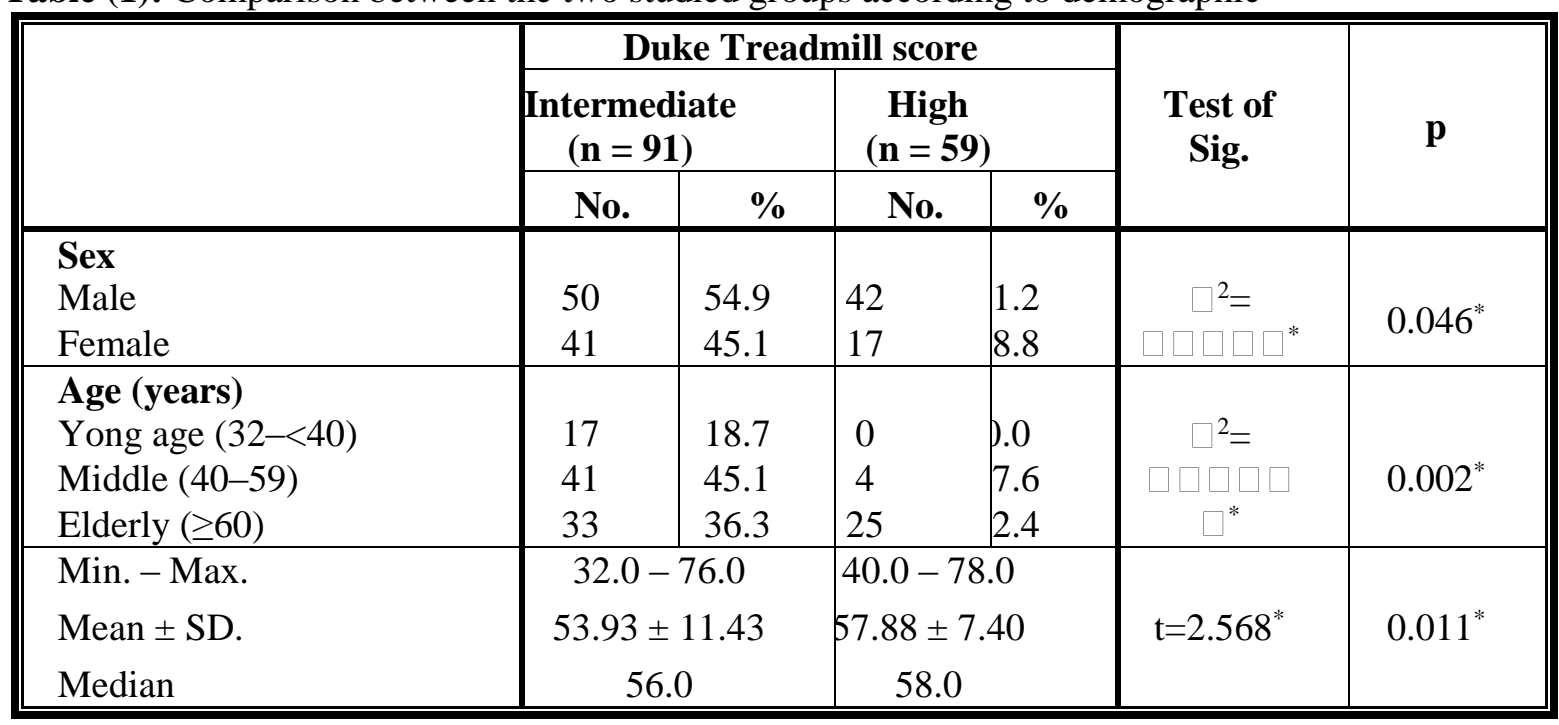

\section{Risk factors}

Regarding diabetes, there was statistically significant difference found between two groups, the p value was 0.005 . Regarding hypertension, there was statistically significant difference found between two groups the $p$ value was 0.042 . Regarding hypercholesterolemia, there was statistically significant difference found between two groups, the $p$ value was 0.039 .

Regarding smoking, there was statistically significant difference found between two groups, the $\mathrm{p}$ value was 0.021 (statistically significant).

Regarding Family history of IHD, there was statistically significant difference found between two groups the $p$ value was 0.020 .

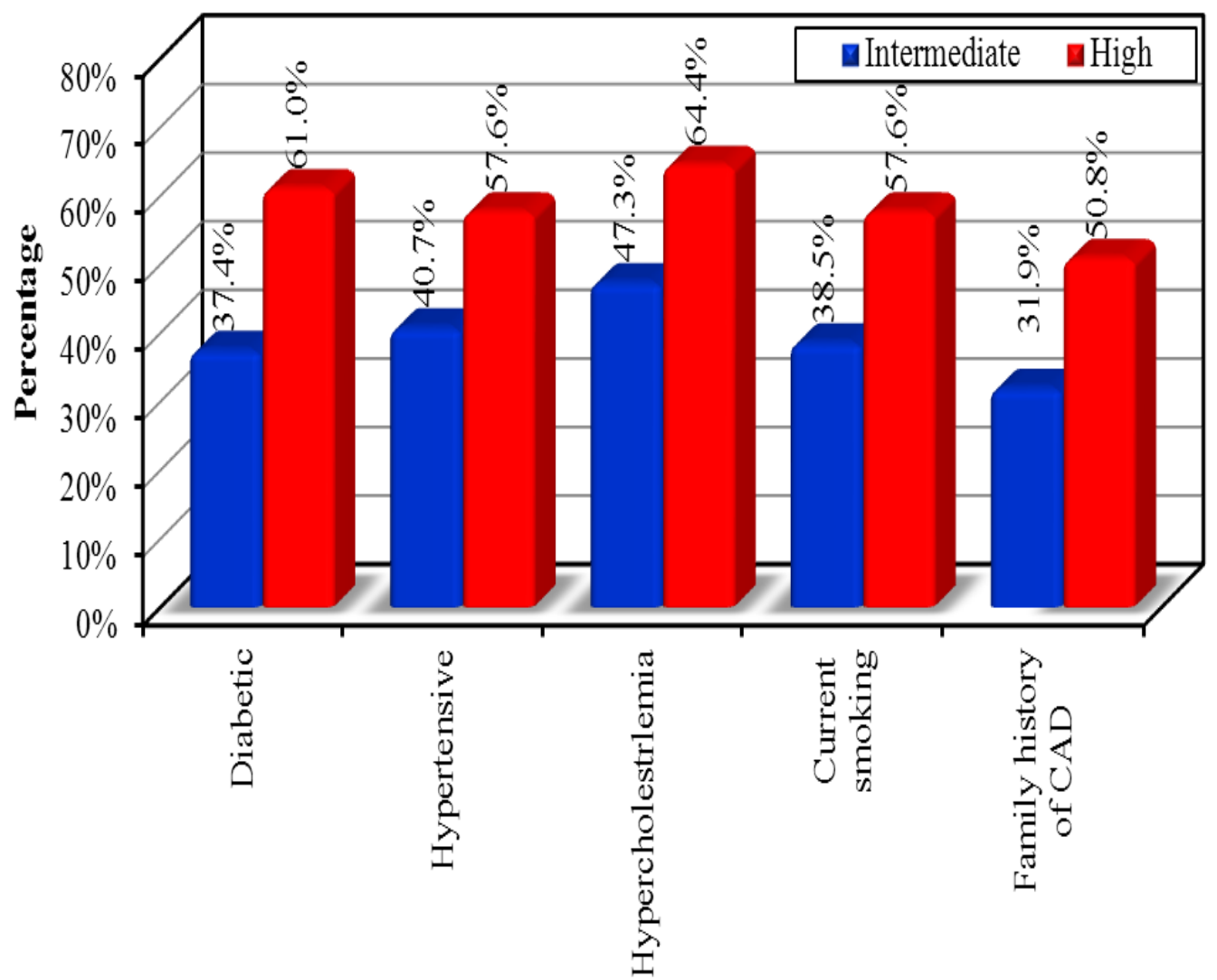

Risk Factors

Figure (1): Percentage of risk factors in two groups 


\section{Treadmill test parameters:}

\section{Treadmill angina}

The percentage of patients with anginal pain during exercise in Group II was $100 \%$ while in group I was $38.5 \%$, the $p$ value was 0.001 (statistically significant).

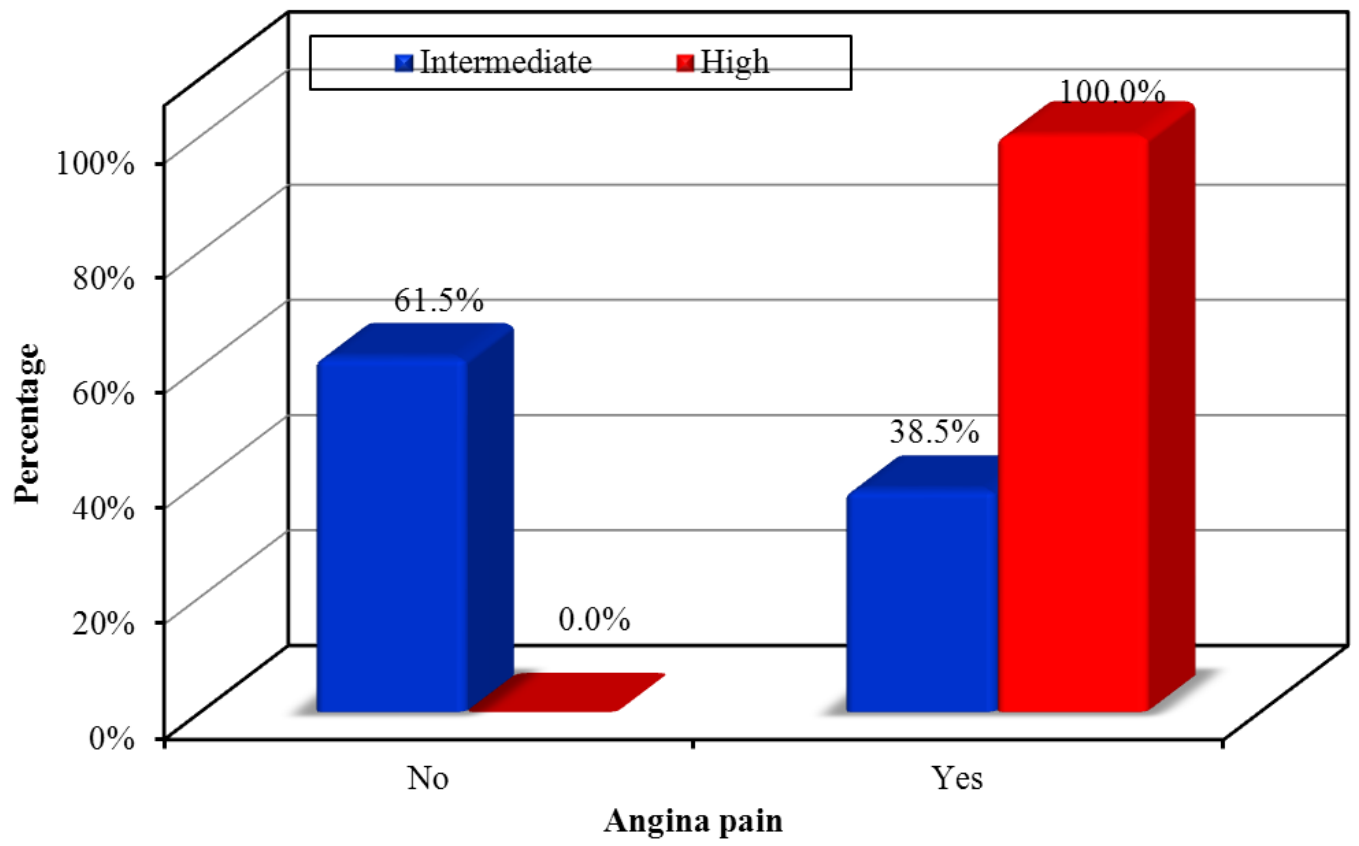

Figure (2): The percentage of patients with and without anginal pain during in two groups.

\section{Type of ST- segment depression:}

For type of ST segment depression; the percentage of patients with ST segment depression down sloping in group II was $62.7 \%$ while in group I was $40.7 \%$, the percentage of patients with ST segment depression horizontal in group II was $37.3 \%$ while in group I was $22 \%$, the p value was $<0.001$ (statistically significant).

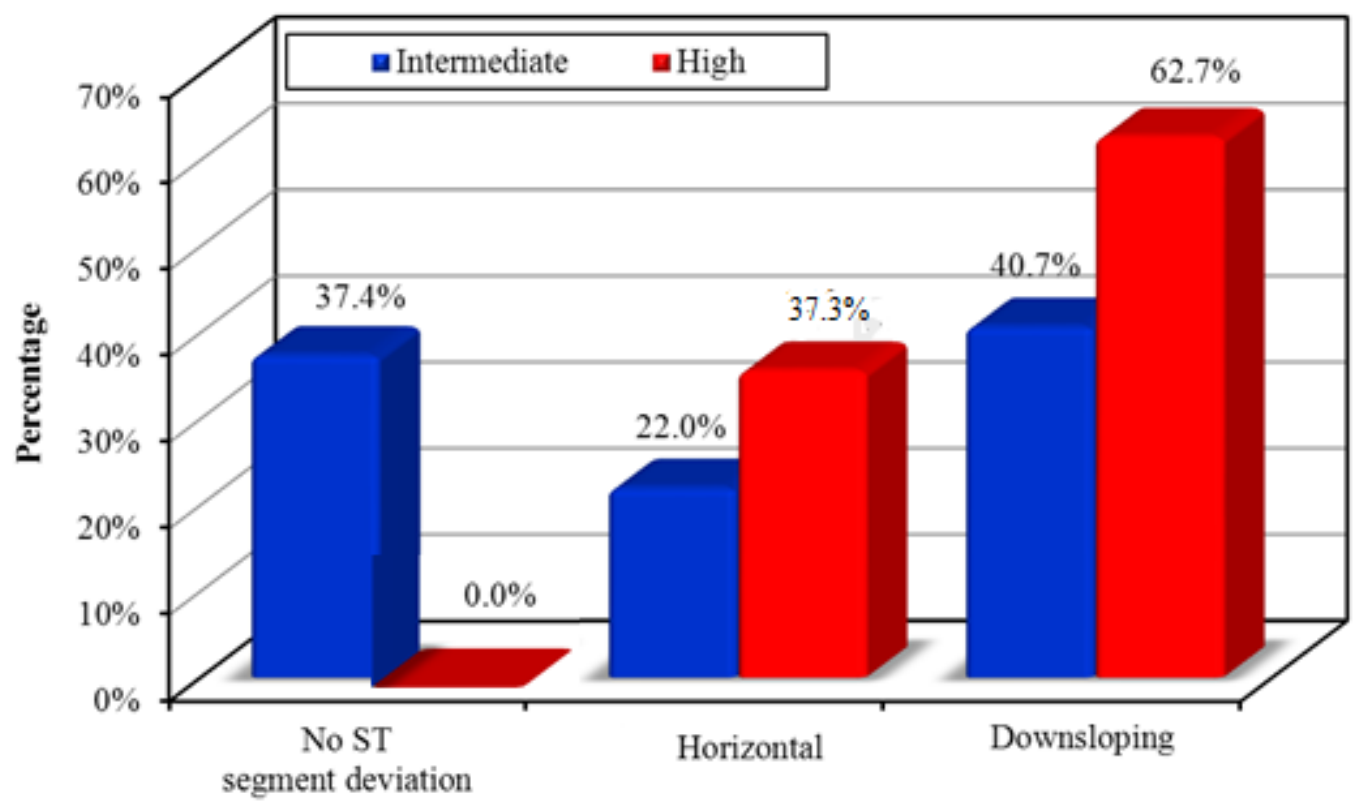

Type of ST segment configuration

Figure (3): Comparison between the two studied groups according to type of ST segment depression

Regarding degree of ST segment depression; mean ST segment depression in group I was 1.55, while in group II was 2.33 , the $\mathrm{p}$ value was 0.001 (statistically significant). 
Table (2): Comparison between the two studied groups according to degree of ST segment deviation

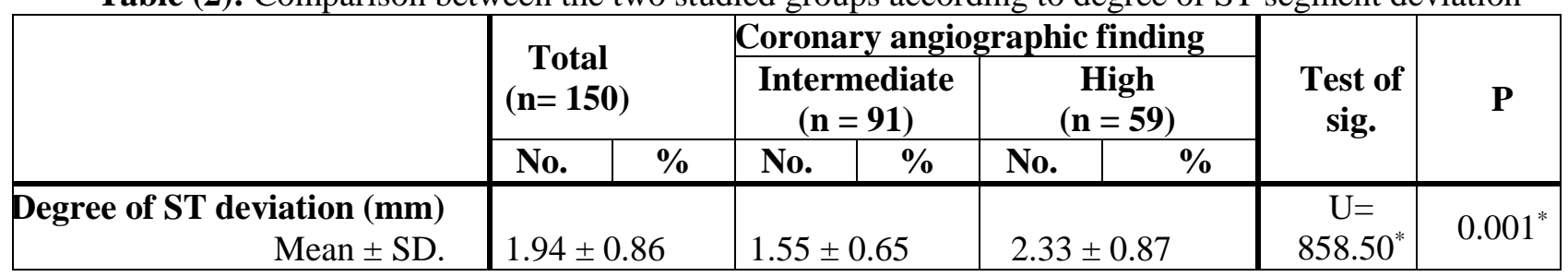

\section{Duke Treadmill score:}

There was significant difference between intermediate and high treadmill score as regarding coronary angiographic findings $(\mathrm{P}=0.032)$.

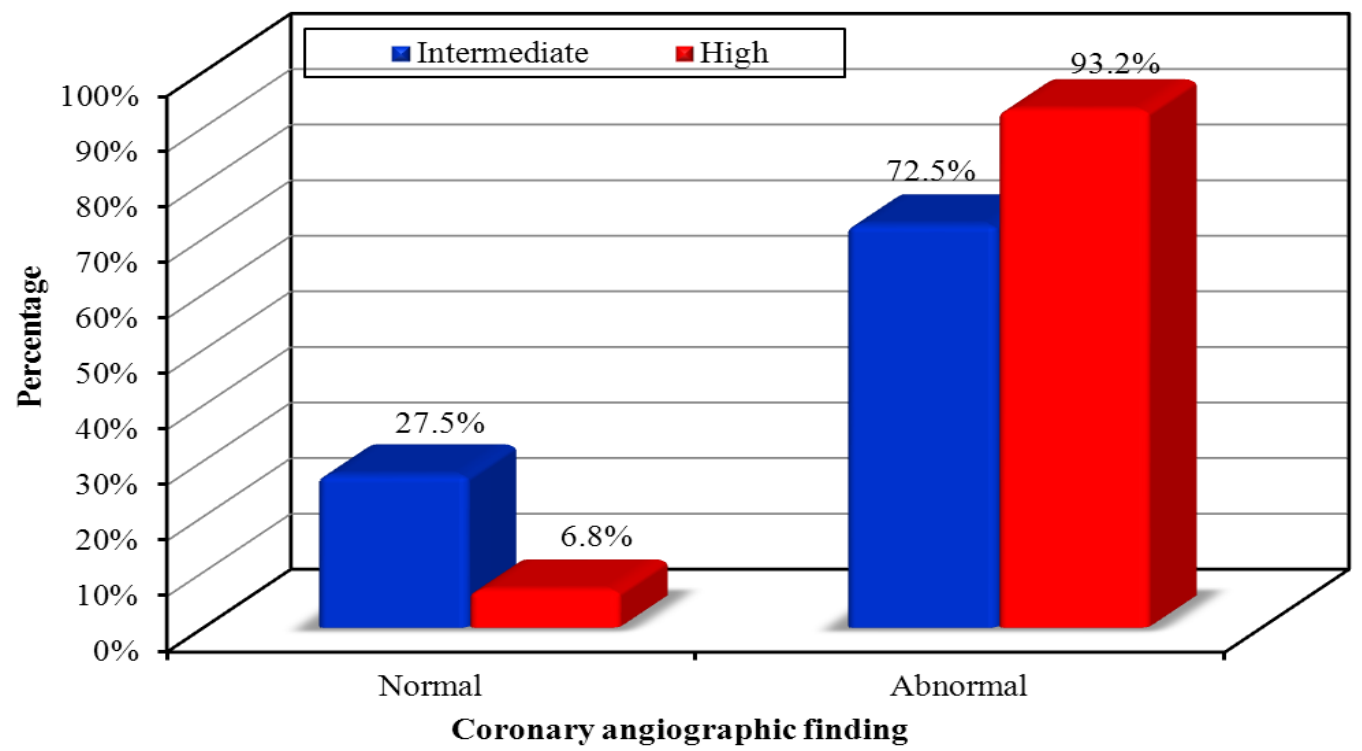

Figure (4): Percentage of normal and abnormal groups in High and Intermediate Duke Treadmill risk score groups

\section{Relationship between number of vessels and treadmill scores;}

The left main stem and three vessels disease occurred more in high risk group (54.2\%) and it was significantly more than intermediate risk group $(18.7 \%)$, while normal and single vessel occurred more in intermediate risk group $(6.8 \%, 11.6 \%)$ and it was significantly more than high risk group ( $\mathrm{P}$ value $<0.001)$.

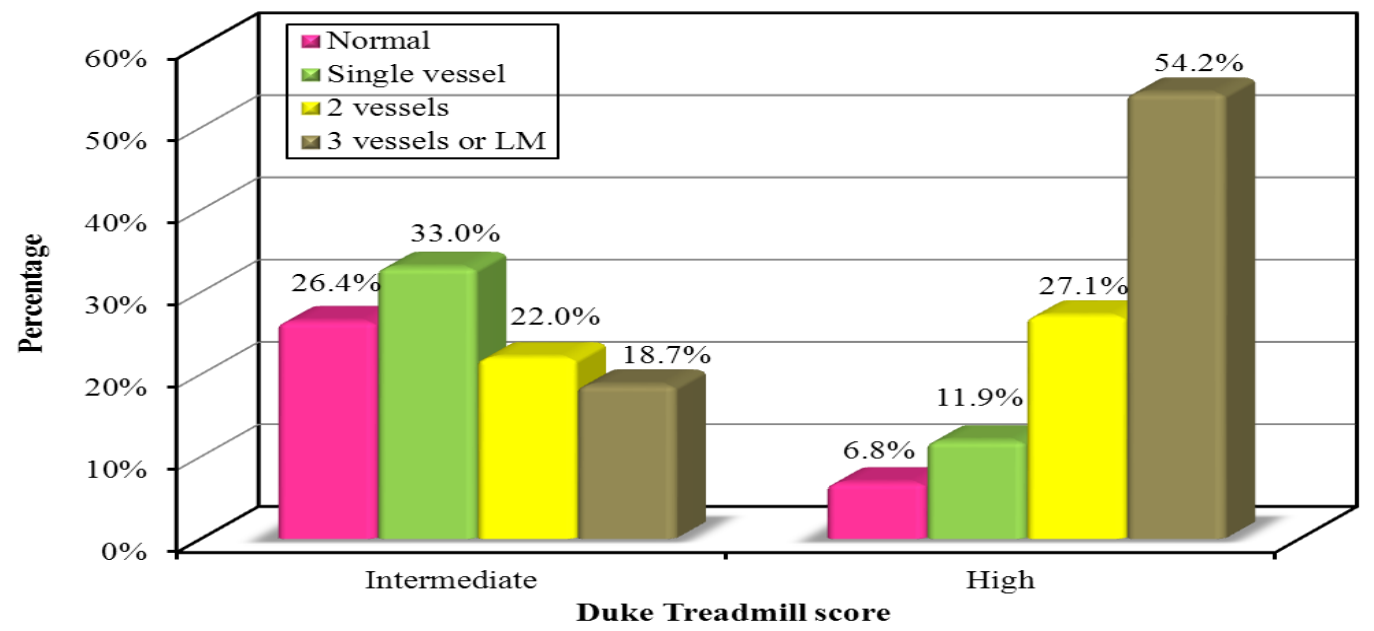

Figure (5): Correlation between number of vessels and treadmill scores.

Intermediate, High risk groups and number of risk factors in relation to results of coronary angiography:

Table(6) shows the treadmill test risk groups and number of risk factors in relation to results of coronary angiography in which the adding of risk factors to Duke's score were statistically significant in intermediate risk group ( $\mathrm{p}$ value 0.001 ), while adding risk factors to high risk group of no value because about (90-100\%) of high risk group had abnormal coronary angiographic result. NB: risk factors include age $\geq 60$ years, Diabetes mellitus, Hypertensive, Hypercholesterolemia, Smoking and Family history of IHD. 
Table (3): Intermediate risk groups and number of risk factors in relation to results of coronary angiography

\begin{tabular}{|c|c|c|c|c|c|c|}
\hline \multirow{3}{*}{$\begin{array}{l}\text { Risk factors in } \\
\text { intermediate and high risk } \\
\text { groups }\end{array}$} & \multicolumn{4}{|c|}{ Coronary angiographic finding } & \multirow{3}{*}{$\square^{\square}$} & \multirow{3}{*}{${ }^{\mathbf{F E}} \mathbf{p}$} \\
\hline & \multicolumn{2}{|c|}{ Normal } & \multicolumn{2}{|c|}{ Abnormal } & & \\
\hline & No. & $\%$ & No. & $\%$ & & \\
\hline Intermediate & \multicolumn{2}{|c|}{$(n=24)$} & \multicolumn{2}{|c|}{$(n=67)$} & & \multirow[b]{3}{*}{$0.001^{*}$} \\
\hline More than 1risk factor & 8 & 33.3 & 56 & 83.6 & $\square \square \square$ & \\
\hline No & 16 & 66.7 & 11 & 16.4 & $\underset{\square}{\square \square}$ & \\
\hline
\end{tabular}

\section{DISCUSSION}

\section{Demographic data:}

Results of our study regarding age showed that the mean age was significantly higher in high risk group than intermediate risk group. So age was found to be an independent predictor of coronary artery disease in our study. This is in agreement with the result of Raxwal $\boldsymbol{e t}$ al. ${ }^{(13)}$ study.

There was statistically significant difference found between two groups according to sex the $\mathrm{p}$ value was 0.002 (statistically significant). This is in agreement with finding of Shaw et al. ${ }^{(14)}$ study.

Detry et al. ${ }^{(15)}$ found that women evaluated for chest pain had less coronary artery disease and did differently on the treadmill from men, which are also in confirm our observation.

\section{Risk factors:}

Regarding hypertension, diabetes mellitus and smoking, we found that, there was significant difference between two groups,. This is in accordance with the result of Shaw et al. ${ }^{(14)}$ study.

Regarding Family history of ischemic heart disease, we found that, there was significant difference between two groups. This is in accordance with the result of Steven ${ }^{(16)}$ study.

Regarding Hypercholesterolemia, we found that, there was significant difference between two groups. This is in accordance with the result of Gabaldo et al. ${ }^{(17)}$ study

\section{Duke Treadmill Score:}

In our results there was significant difference between moderate and high risk treadmill scores regarding coronary angiography. It was found that over 93.2\% of cases with high risk had significant coronary disease compared with $72.6 \%$ of cases with moderate risk had significant coronary disease. This in agreement with our findings Alexander et $\boldsymbol{a l} .{ }^{(\mathbf{1 8})}$. But in our study we excluded patients with low risk duke treadmill score as according to recent stable coronary artery disease guidelines, management of this group with non-invasive strategy $^{(19)}$.

We found that Duke's treadmill score is valuable in diagnosing stable coronary artery disease specially high risk group, as The left main stem and three vessels disease occurred more in high risk group (54.2\%) and it was significantly more than intermediate risk group (18.7\%), while normal and single vessel occurred more in intermediate risk group $(26.4 \%, 33 \%)$ and it was significantly more than high risk group. (P value $<0.001$ ) in accordance with Shaw et $\boldsymbol{a l} .{ }^{(14)}$ and Peters et $\boldsymbol{a l} .{ }^{(20)}$ studies.

This study revealed that $26.4 \%$ of patients with intermediate risk Duke's score had normal coronary angiographic results and this may be due to minor vessels disease or false positive results of exercise ECG stress test or under estimation of severity of the coronary lesion. The false positive result of exercise test may be due to false result of test (positive result in normal coronary vessels) or false result of coronary angiography (as in patient with distal vessels disease) ${ }^{(\mathbf{2 1 )}}$.

Since the overall sensitivity of exercise stress electrocardiography is $68 \%$, a negative result doesn't exclude coronary artery disease, but it makes the possibility of multi-vessel or left main coronary artery disease very rare.

We tried to add risk factors to increase sensitivity of Duke's treadmill score to suspect coronary artery disease. The results showed that analysis of risk factors was of value in patients with intermediate risk group, and so we suggest to take risk factors in consideration before performing coronary angiography in patients with intermediate risk group ( 8 patients out of 64 patients with intermediate risk group whom had risk factors appeared to have normal coronary angiography, while 16 patients out of 27 patients with intermediate risk and no risk factors appeared to have normal coronary angiography). Adding of risk factors to high risk group had no benefit as most of them had abnormal coronary angiographic results.

\section{CONCLUSIONS}

Exercise ECG stress testing remains a good test in predicting the significance of coronary artery stenosis. The Duke Treadmill Score (DTS) is a good predictor for coronary artery disease.

High risk DTS group should be referred immediately to coronary angiography as high risk level has been associated with multi-vessel and LMCA than intermediate risk DTS group. Intermediate risk Duke Treadmill Score group exercise test results should be managed individually according to clinical data and type of ST-Segment depression during exercise ECG stress testing. Coronary angiography remains the golden test for assessment of CAD. 


\section{REFERENCES}

1. Libby P, Ridker PM, Hansson GKet al. (2009): Transatlantic Network on Atherothrombosis. Inflammation in atherosclerosis: from pathophysiology to practice. J Am Coll Cardiol., 54: 2129-2138.

2. Kręcki R, Arazińska A, Peruga JZ et al. (2014): Characteristics, management and five-year outcomes of patients with high risk, stable multivessel coronary heart disease. Kardiol Pol., 72: 262-268.

3. Hansson GK (2005): Inflammation, atherosclerosis, and coronary artery disease. N Engl J Med., 352: 1685-1695.

4. Muller JE, Ludmer PL, Willich SN et al.(1987):Circadian variation in the frequency of sudden cardiac death. Circulation, 75: 131-8.

5. L'Abbate A (1986): Rationale Sequence toward the diagnosis of myocardial ischemia. Can J Cardiol.,11: 233-41.

6. Fletcher GF, Balady GJ, Froelicher VF et al. (1995).: Exercise standards. A statement for healthcare professionals from the American Heart Association.Writing Group. Circulation, 91: 580-585.

7. Laupacis A, Sekar N and Stiell I (1997): Clinical prediction rulesa review and suggested modifications of methodologic standards. JAMA., 277:488-494.

8. Mark DB, Shaw L, Harrell FE et al. (1991): Prognostic value of a treadmill exercise score in outpatients with suspected coronary artery disease. N Engl J Med., 325(12): 849-55.

9. Yamada H, Do D, Morise A et al. (1997): Review of studies utilizing multi-variable analysis of clinical and exercise test data to predict angiographic coronary artery disease. Prog Cardiovasc Dis., 39:457-481.

10. Fearon WF1, Gauri AJ, Myers J et al. (2002): A comparison of treadmill scores to diagnose coronary artery disease. Clin Cardiol., 25:117.
11. Fletcher GF, Flipse TR, Kligfield $P$ et al.(1998): Current status of ECG stress testing. Curr Probl Cardiol., 23: 253.

12. Miranda CP, Liu J, Kadar A et al. (1992): Usefulness of exercise-induced ST-segment depression in the inferior leads during exercise testing as a marker for coronary artery disease. Am J Cardiol., 69(4): 303-307

13. Raxwal V, Shetler K, Morise A et al. (2001): Simple treadmill score to diagnose coronary artery disease. Chest, 119:1933-1940.

14. Shaw LJ, Peterson ED, Shaw LK et al. (1998): Use of a prognostic treadmill score in identifying diagnostic coronary disease subgroups. Circulation, 98:1622-1630.

15. Detry JR, Kapita MB andCosyns J (1997): Diagnostic value of history and maximal exercise electrocardiography in men and women suspected of coronary heart disease. Circulation, 56:756-761.

16. Steven $\mathbf{S}(\mathbf{1 9 8 4})$ :Family history as an independent risk factor for coronary artery disease. JACC., 4(4):793-801.

17. Gabaldo K, Hadzibegović I, Prvulović D et al. (2008): Duke Treadmill Score in prioritizing patients for coronary angiography: Retrospective study of a Croatian regional hospital. CollAntropol., 32:375-380.

18. Alexander KP, Shaw LJ, Shaw LK et al. (1998): Value of exercise treadmill testing in Women. J Am CollCardiol., 32:1657-1664.

19. Montalescot G, Sechtem U (2013): ESC guidelines on the management of stable coronary artery disease The Task Force on the management of stable coronary artery disease of the European Society. Eur Hear., 34(38):29493003.

20. Peters RM, Peters JC andShanies SA (2000):Predicting extent of coronary disease: fuzzy cluster analysis vs. Duke treadmill score. J Clin Basic Cardiol., 3: 39-42.

21. Weinsaft JW, Wong FJ, Walden J et al. (1991): Anatomic distribution of myocardial ischemia as a determinant of exercise-induced ST-segment depression. Am J Cardiol., 68:569-574. 